\title{
Positive Normalization of Discrete Descriptor System under Disturbance
}

\author{
Ahmad Iqbal Baqi, Admi Nazra, Zulakmal, Lyra Yulianti and Muhafzan* \\ Department of Mathematics, Faculty of Mathematics and Natural Sciences \\ Andalas University, 25163 Kampus Unand Limau Manis, Padang, Indonesia \\ *Corresponding author: muhafzan@sci.unand.ac.id
}

Article history

Received: 30 August 2018

Received in revised form: 16 December 2018

Accepted: 17 December 2018

Published on line: 31 December 2018

\begin{abstract}
It is well known the descriptor systems have a wide application field. Usually it appear as a mathematical model of a real problem, mainly the model that involves the input output relationship. It is well known that a descriptor linear system has an unique solution if the pencil matrix of the system is regular. However, there are some systems that are not regular. Moreover, even though the system is regular the solution can contain the noncausal behavior. Therefore, it is necessary to normalize the descriptor system so as it has well behavior. In this paper, we propose a feedback to normalize a discrete descriptor system under disturbance. Furthermore, we establish a sufficient condition in order for the discrete descriptor system under disturbance can be normalized positively.
\end{abstract}

Keywords Normalization; discrete descriptor system; disturbance.

Mathematics Subject Classification 39A06, 93C55.

\section{Introduction}

Let us consider the following linear discrete descriptor system under disturbance

$$
\begin{aligned}
\mathcal{E} \mathbf{x}_{i+1} & =\mathcal{A} \mathbf{x}_{i}+\mathcal{B} \mathbf{u}_{i}+\mathcal{C} \boldsymbol{\omega}_{i}, i \in \mathbb{Z}_{+} \\
\mathbf{y}_{i} & =\mathcal{D} \mathbf{x}_{i}
\end{aligned}
$$

where $\mathbf{x}_{i} \in \mathbb{R}^{n}$ is the state, $\mathbf{y}_{i} \in \mathbb{R}^{p}$ is the output, $\mathbf{u}_{i} \in \mathbb{R}^{m}$ is the control, $\boldsymbol{\omega}_{i} \in \mathbb{R}^{q}$ is the disturbance, $\mathcal{E}, \mathcal{A} \in \mathbb{R}^{n \times n}, \mathcal{B} \in \mathbb{R}^{n \times m}, \mathcal{C} \in \mathbb{R}^{n \times q}, \mathcal{D} \in \mathbb{R}^{p \times n}, \mathbb{Z}_{+}$is the set of nonnegative integer number and $\operatorname{rank}(\mathcal{E})=r<n$. It is well-known if the system (1) is regular, namely $\operatorname{det}(\gamma \mathcal{E}-\mathcal{A}) \neq 0$ for some $\gamma \in \mathbb{C}$, the system (1) has an unique solution. As shown in [1], any regular pencil of index $\nu$ can be reduced in to the following Cronecker form

$$
Q_{1}(\gamma \mathcal{E}-\mathcal{A}) P_{1}=\gamma\left[\begin{array}{cc}
I_{r} & O \\
O & N
\end{array}\right]-\left[\begin{array}{cc}
J & O \\
O & I_{n-r}
\end{array}\right]
$$


for some nonsingular matrices $Q_{1}, P_{1} \in \mathbb{R}^{n \times n}$ where $J \in \mathbb{R}^{r \times r}$ is a Jordan Canonic form and $N \in \mathbb{R}^{(n-r) \times(n-r)}$ is a Nilpotent matrix. Therefore, under transformation

$$
\mathbf{q}=P_{1}^{-1} \mathbf{x}=\left[\begin{array}{l}
\mathbf{q}_{1} \\
\mathbf{q}_{2}
\end{array}\right]
$$

the system (1) can be written as

$$
\begin{aligned}
\mathbf{q}_{1, i+1} & =J \mathbf{q}_{1, i}+B_{1} \mathbf{u}_{i}+C_{1} \boldsymbol{\omega}_{i} \\
N \mathbf{q}_{2, i+1} & =\mathbf{q}_{2, i}+B_{2} \mathbf{u}_{i}+C_{2} \boldsymbol{\omega}_{i} \\
\mathbf{y}_{i} & =D_{1} \mathbf{q}_{1, i}+D_{2} \mathbf{q}_{2, i},
\end{aligned}
$$

where

$$
\left[\begin{array}{l}
B_{1} \\
B_{2}
\end{array}\right]=Q_{1} \mathcal{B}, \quad\left[\begin{array}{l}
C_{1} \\
C_{2}
\end{array}\right]=Q_{1} \mathcal{C} \text { and }\left[\begin{array}{cc}
D_{1} & D_{2}
\end{array}\right]=\mathcal{D} P_{1},
$$

with $B_{1} \in \mathbb{R}^{r \times m}, C_{1} \in \mathbb{R}^{r \times q}, B_{2} \in \mathbb{R}^{(n-r) \times m}, C_{2} \in \mathbb{R}^{(n-r) \times q}, D_{1} \in \mathbb{R}^{p \times r}, D_{2} \in \mathbb{R}^{p \times(n-r)}$. The solution of (4) is given by [1]

$$
\begin{aligned}
& \mathbf{q}_{1, i}=J^{i} \mathbf{q}_{1,0}+\sum_{j=0}^{i-1} J^{i-j-1}\left(B_{1, i} \mathbf{u}_{i}+C_{1} \boldsymbol{\omega}_{i}\right) \\
& \mathbf{q}_{2, i}=-\sum_{j=i}^{\nu-1} N^{j}\left(B_{2, i} \mathbf{u}_{i}+C_{2} \boldsymbol{\omega}_{i}\right)
\end{aligned}
$$

where $\nu=\operatorname{ind}(\mathcal{E})$. Different to the normal system, i.e. in the case $\mathcal{E}=I$, the discrete desciptor system may posses noncausal behavior, i.e. the solution may depend on future values of the sequences $\mathbf{q}_{i}, \mathbf{u}_{i}, \boldsymbol{\omega}_{i}$. This behavior is shown by (6). This noncausal behavior constitutes an unwanted behaviour because it may cause degradation in performance or even destroy the system. Therefore, it is important to eliminate this noncausal behavior. One of the way for eliminating the noncausal behavior of descriptor system is by normalization. As stated in [2] and [3] that the noncausal behavior of the system (1) can be eliminated using a state feedback of the form $\mathbf{u}_{i}=-\mathcal{K} \mathbf{x}_{i}, i \in \mathbb{Z}_{+}$, and the sufficient and necessary condition for freenes the noncausal behavior of the system $(1)$ is $\operatorname{rank}\left[\begin{array}{ll}\mathcal{E} & \mathcal{B}\end{array}\right]=n$.

In the application fields in which the system (1) appears as a model of some real problems, the non-negativeness of the state variable is a must. The system (1) without disturbance is called positive if for all $i \in \mathbb{Z}_{+}$we have $\mathbf{x}_{i} \in \mathbb{R}_{+}^{n}$ and $\mathbf{y}_{i} \in \mathbb{R}_{+}^{p}$ for any control $\mathbf{u}_{i} \in \mathbb{R}_{+}^{m}$ [4]. For the normal system, the positiveness is defined similarly. It well known that if $\operatorname{rank}(\mathcal{E})=n$, system (1) without disturbance is positive iff $\mathcal{E}^{-1} \mathcal{A} \in \mathbb{R}_{+}^{n \times n}, \mathcal{E}^{-1} \mathcal{B} \in \mathbb{R}_{+}^{n \times m}$ and $\mathcal{D} \in \mathbb{R}_{+}^{p \times n}$. In this paper we investigate the problem of positive normalization for descriptor system (1) using the difference output feedback such that the resultant closed loop system is positive. Furthermore, we establish a sufficient condition in order for the discrete descriptor system under disturbance can be normalized positively. 


\section{Problem Formulation}

We are interested in the problem of positive normalization for descriptor system (1) using the following difference output feedback

$$
\mathbf{u}_{i}=-\mathcal{K}\left(\mathbf{y}_{i+1}-\mathbf{y}_{i}\right)
$$

where $\mathcal{K} \in \mathbb{R}^{m \times p}$ is the gain matrix to be determined. Applying the feedback (7) to the system $(1)$, it is obtained the following resultant closed loop system:

$$
(\mathcal{E}+\mathcal{B K} \mathcal{K}) \mathbf{x}_{i+1}=(\mathcal{A}+\mathcal{B} \mathcal{K} \mathcal{D}) \mathbf{x}_{i}+\mathcal{C} \boldsymbol{\omega}_{i}
$$

Definition 1 System (1) is called normalizable positively if a difference feedback controller (7) can be found such that the resulted closed loop system (8) is normal, i.e.

$$
\operatorname{det}(\mathcal{E}+\mathcal{B K} \mathcal{D}) \neq 0
$$

and the system (8) is positive.

It is obvious that if the matrix $\operatorname{det}(\mathcal{E}+\mathcal{B K} \mathcal{D}) \neq 0$ then the system (1) is noncausal. Consequently, our task now is to establish the criteria to ensure the system (1) is noncausal and the resultant closed loop system (8) to be positive under the assumption $\mathcal{C} \in \mathbb{R}_{+}^{n \times q}$ and $\boldsymbol{\omega}_{i} \in \mathbb{R}_{+}^{q}, i \in \mathbb{Z}_{+}$.

To fulfill our task, we need the following results.

Definition $2 A$ matrix $\mathcal{A} \in \mathbb{R}^{n \times n}$ is said to be monotone if it is non-singular and $\mathcal{A}^{-1} \in$ $\mathbb{R}_{+}^{n \times n}$. A matrix $\mathcal{M} \in \mathbb{R}_{+}^{n \times n}$ is said a monomial matrix if in every row and column of $\mathcal{M}$ there is exactly one nonzero entry [5].

It has been proved in [5] that $\mathcal{A}^{-1} \in \mathbb{R}_{+}^{n \times n}$ if and only if $\mathcal{A}$ is monomial.

\section{Definition 3 [5]}

1. Two matrices $\mathcal{M} \in \mathbb{R}_{+}^{n \times n}$ and $\mathcal{S} \in \mathbb{R}_{+}^{n \times n}$ are said positively equivalent if there exist two monomial matrices $P$ and $Q$ such that $\mathcal{S}=Q \mathcal{M} P$.

2. A matrix $\mathcal{M} \in \mathbb{R}_{+}^{n \times n}$ with $\operatorname{rank}(\mathcal{M})=s$ is said $s-$ monomial if it is positively equivalent to the matrix $\operatorname{diag}\left(\mathcal{M}_{s}, O\right)$, where $\mathcal{M}_{s}$ is monomial.

The $s$-monomial matrices have the following properties.

\section{Theorem 1 [5]}

1. The matrix $\mathcal{M}$ is $s$-monomial iff $\mathcal{M}$ has $n-s$ rows and columns with all entries equal to zero and s rows and columns with only one entry different to zero.

2. If the matrix $\mathcal{M}$ is $s-$ monomial, then it is positively equivalent to the matrix $\operatorname{diag}\left(I_{s}, O\right)$. 


\section{Main Result}

First, let us generate the matrix $\mathcal{K} \in \mathbb{R}^{m \times p}$ such that $\operatorname{det}(\mathcal{E}+\mathcal{B K} \mathcal{D}) \neq 0$. Assume that the matrix $\mathcal{E}$ is positively equivalent to $s$-monomial matrix $\mathcal{M}$, that is

$$
Q \mathcal{E} P=\left[\begin{array}{cc}
\mathcal{M}_{s} & O \\
O & O
\end{array}\right]
$$

for some monomial matrices $Q$ and $P$. Moreover, let

$$
Q \mathcal{A} P=\left[\begin{array}{cc}
\mathcal{A}_{1} & \mathcal{A}_{2} \\
\mathcal{A}_{3} & \mathcal{A}_{4}
\end{array}\right], Q \mathcal{B}=\left[\begin{array}{c}
\mathcal{B}_{1} \\
\mathcal{B}_{2}
\end{array}\right] \text { and } \mathcal{D} P=\left[\begin{array}{ll}
O & \mathcal{D}_{2}
\end{array}\right]
$$

where $\mathcal{A}_{1} \in \mathbb{R}^{s \times s}, \mathcal{A}_{2} \in \mathbb{R}^{s \times(n-s)}, \mathcal{A}_{3} \in \mathbb{R}^{(n-s) \times s}, \mathcal{A}_{4} \in \mathbb{R}^{(n-s) \times(n-s)}, \mathcal{B}_{1} \in \mathbb{R}^{s \times m}, \mathcal{B}_{2} \in \mathbb{R}^{(n-s) \times m}, \mathcal{D}_{2} \in$ $\mathbb{R}^{p \times(n-s)} \quad$ Assume that $\mathcal{D}_{2}$ has full rank. The condition $\operatorname{rank}\left[\begin{array}{ll}\mathcal{E} & \mathcal{B}\end{array}\right]=n$ is equivalent to $\mathcal{B}_{2}$ has full row rank. Choose $\mathcal{K}=\left(\mathcal{D}_{2} \mathcal{B}_{2}\right)^{\top}$, we have

$$
\begin{aligned}
\mathcal{E}+\mathcal{B K D} & =Q^{-1}\left[\begin{array}{cc}
\mathcal{M}_{s} & O \\
O & O
\end{array}\right] P^{-1}+Q^{-1} Q \mathcal{B} \mathcal{K} \mathcal{D} P P^{-1} \\
& =Q^{-1}\left(\left[\begin{array}{cc}
\mathcal{M}_{s} & O \\
O & O
\end{array}\right]+\left[\begin{array}{l}
\mathcal{B}_{1} \\
\mathcal{B}_{2}
\end{array}\right] \mathcal{B}_{2}^{\top} \mathcal{D}_{2}^{\top}\left[\begin{array}{ll}
O & \mathcal{D}_{2}
\end{array}\right]\right) P^{-1} \\
& =Q^{-1}\left(\left[\begin{array}{cc}
\mathcal{M}_{s} & O \\
O & O
\end{array}\right]+\left[\begin{array}{cc}
O & \mathcal{B}_{1} \mathcal{B}_{2}^{\top} \mathcal{D}_{2}^{\top} \mathcal{D}_{2} \\
O & \mathcal{B}_{2} \mathcal{B}_{2}^{\top} \mathcal{D}_{2}^{\top} \mathcal{D}_{2}
\end{array}\right]\right) P^{-1} \\
& =Q^{-1}\left[\begin{array}{cc}
\mathcal{M}_{s} & \mathcal{B}_{1} \mathcal{B}_{2}^{\top} \mathcal{D}_{2}^{\top} \mathcal{D}_{2} \\
O & \mathcal{B}_{2} \mathcal{B}_{2}^{\top} \mathcal{D}_{2}^{\top} \mathcal{D}_{2}
\end{array}\right] P^{-1} .
\end{aligned}
$$

Since $\mathcal{B}_{2}$ and $\mathcal{D}_{2}$ have full rank, the matrix $\mathcal{B}_{2} \mathcal{B}_{2}^{\top} \mathcal{D}_{2}^{\top} \mathcal{D}_{2}$ is nonsingular. This $\operatorname{implies} \operatorname{det}(\mathcal{E}+$ $\mathcal{B K} \mathcal{D}) \neq 0$.

Furthermore, we construct the conditions such that the matrix $(\mathcal{E}+\mathcal{B K} \mathcal{D})^{-1} \in \mathbb{R}_{+}^{n \times n}$ and $(\mathcal{A}+\mathcal{B K} \mathcal{D}) \in \mathbb{R}_{+}^{n \times n}$. Note that

$$
\begin{aligned}
(\mathcal{E}+\mathcal{B K} \mathcal{L})^{-1} & =P\left[\begin{array}{cc}
\mathcal{M}_{s} & \mathcal{B}_{1} \mathcal{B}_{2}^{\top} \mathcal{D}_{2}^{\top} \mathcal{D}_{2} \\
O & \mathcal{B}_{2} \mathcal{B}_{2}^{\top} \mathcal{D}_{2}^{\top} \mathcal{D}_{2}
\end{array}\right]^{-1} Q \\
& =P\left[\begin{array}{cc}
\mathcal{M}_{s}^{-1} & -\mathcal{M}_{s}^{-1} \mathcal{B}_{1} \mathcal{B}_{2}^{\top} \mathcal{D}_{2}^{\top} \mathcal{D}_{2}\left(\mathcal{B}_{2} \mathcal{B}_{2}^{\top} \mathcal{D}_{2}^{\top} \mathcal{D}_{2}\right)^{-1} \\
O & \left(\mathcal{B}_{2} \mathcal{B}_{2}^{\top} \mathcal{D}_{2}^{\top} \mathcal{D}_{2}\right)^{-1}
\end{array}\right] Q \\
& =P\left[\begin{array}{cc}
\mathcal{M}_{s}^{-1} & O \\
O & I
\end{array}\right]\left[\begin{array}{cc}
I & -\mathcal{B}_{1} \mathcal{B}_{2}^{\top} \mathcal{D}_{2}^{\top} \mathcal{D}_{2}\left(\mathcal{B}_{2} \mathcal{B}_{2}^{\top} \mathcal{D}_{2}^{\top} \mathcal{D}_{2}\right)^{-1} \\
O & \left(\mathcal{B}_{2} \mathcal{B}_{2}^{\top} \mathcal{D}_{2}^{\top} \mathcal{D}_{2}\right)^{-1}
\end{array}\right] Q .
\end{aligned}
$$

It is obvious that $\mathcal{M}_{s}^{-1} \in \mathbb{R}_{+}^{s \times s}$ (based on the Definition 3) and one can see that if $\left(\mathcal{B}_{2} \mathcal{B}_{2}^{\top} \mathcal{D}_{2}^{\top} \mathcal{D}_{2}\right)^{-1} \in$ $\mathbb{R}_{+}^{(n-s) \times(n-s)}$ and $\mathcal{B}_{1} \mathcal{B}_{2}^{\top} \mathcal{D}_{2}^{\top} \mathcal{D}_{2} \in \mathbb{R}_{-}^{s \times(n-s)}$, then $(\mathcal{E}+\mathcal{B K} \mathcal{D})^{-1} \in \mathbb{R}_{+}^{n \times n}$. 
Moreover,

$$
\begin{aligned}
\mathcal{A}+\mathcal{B K} \mathcal{D} & =Q^{-1}\left[\begin{array}{cc}
\mathcal{A}_{1} & \mathcal{A}_{2} \\
\mathcal{A}_{3} & \mathcal{A}_{4}
\end{array}\right] P^{-1}+Q^{-1} Q \mathcal{B K} \mathcal{D} P P^{-1} \\
& =Q^{-1}\left(\left[\begin{array}{ll}
\mathcal{A}_{1} & \mathcal{A}_{2} \\
\mathcal{A}_{3} & \mathcal{A}_{4}
\end{array}\right]+\left[\begin{array}{c}
\mathcal{B}_{1} \\
\mathcal{B}_{2}
\end{array}\right] \mathcal{B}_{2}^{\top} \mathcal{D}_{2}^{\top}\left[\begin{array}{ll}
O & \mathcal{D}_{2}
\end{array}\right]\right) P^{-1} \\
& =Q^{-1}\left(\left[\begin{array}{cc}
\mathcal{A}_{1} & \mathcal{A}_{2} \\
\mathcal{A}_{3} & \mathcal{A}_{4}
\end{array}\right]+\left[\begin{array}{cc}
O & \mathcal{B}_{1} \mathcal{B}_{2}^{\top} \mathcal{D}_{2}^{\top} \mathcal{D}_{2} \\
O & \mathcal{B}_{2} \mathcal{B}_{2}^{\top} \mathcal{D}_{2}^{\top} \mathcal{D}_{2}
\end{array}\right]\right) P^{-1} \\
& =Q^{-1}\left[\begin{array}{cc}
\mathcal{A}_{1} & \mathcal{A}_{2}+\mathcal{B}_{1} \mathcal{B}_{2}^{\top} \mathcal{D}_{2}^{\top} \mathcal{D}_{2} \\
\mathcal{A}_{3} & \mathcal{A}_{4}+\mathcal{B}_{2} \mathcal{B}_{2}^{\top} \mathcal{D}_{2}^{\top} \mathcal{D}_{2}
\end{array}\right] P^{-1}
\end{aligned}
$$

Once more we can see that if $Q \mathcal{A} P \in \mathbb{R}_{+}^{n \times n}$ such that $\left(\mathcal{A}_{2}+\mathcal{B}_{1} \mathcal{B}_{2}^{\top} \mathcal{D}_{2}^{\top} \mathcal{D}_{2}\right) \in \mathbb{R}_{+}^{s \times(n-s)}$ and $\left(\mathcal{A}_{4}+\mathcal{B}_{2} \mathcal{B}_{2}^{\top} \mathcal{D}_{2}^{\top} \mathcal{D}_{2}\right) \in \mathbb{R}_{+}^{s \times(n-s)}$, then $(\mathcal{A}+\mathcal{B} \mathcal{K} \mathcal{D}) \in \mathbb{R}_{+}^{n \times n}$. Thus we have proved the following Theorem that constitutes the main result of this paper. Compare with the results in [6].

Theorem 2 Consider the system (1) with $\mathcal{E}, \mathcal{A}, \mathcal{B}$ and $\mathcal{D}$ are given in (9) and (10) where $\mathcal{D}_{2}$ have full rank. Then the system of (1) can be normalized positively if the following conditions hold:

1. $\left(\mathcal{B}_{2} \mathcal{B}_{2}^{\top} \mathcal{D}_{2}^{\top} \mathcal{D}_{2}\right)^{-1} \in \mathbb{R}_{+}^{(n-s) \times(n-s)}$

2. $\mathcal{B}_{1} \mathcal{B}_{2}^{\top} \mathcal{D}_{2}^{\top} \mathcal{D}_{2} \in \mathbb{R}_{-}^{s \times(n-s)}$

3. $Q \mathcal{A} P \in \mathbb{R}_{+}^{n \times n}$ such that $\left(\mathcal{A}_{2}+\mathcal{B}_{1} \mathcal{B}_{2}^{\top} \mathcal{D}_{2}^{\top} \mathcal{D}_{2}\right) \in \mathbb{R}_{+}^{s \times(n-s)}$ and $\left(\mathcal{A}_{4}+\mathcal{B}_{2} \mathcal{B}_{2}^{\top} \mathcal{D}_{2}^{\top} \mathcal{D}_{2}\right) \in \mathbb{R}_{+}^{(n-s) \times(n-s)}$

4. $\mathcal{C} \in \mathbb{R}_{+}^{n \times q}$.

As an illustration, let us consider the system (1) where

$$
\mathcal{E}=\left[\begin{array}{lllll}
2 & 0 & 0 & 0 & 0 \\
0 & 1 & 0 & 0 & 0 \\
0 & 0 & 0 & 0 & 0 \\
0 & 0 & 4 & 0 & 0 \\
0 & 0 & 0 & 0 & 0
\end{array}\right], \mathcal{A}=\left[\begin{array}{lllll}
6 & 3 & 2 & 1 & 1 \\
3 & 1 & 2 & 1 & 1 \\
1 & 1 & 0 & 4 & 1 \\
1 & 1 & 5 & 1 & 1 \\
1 & 1 & 3 & 0 & 1
\end{array}\right], \mathcal{B}=\left[\begin{array}{cc}
-1 / 2 & 0 \\
0 & 0 \\
0 & 1 \\
0 & 0 \\
2 & 0
\end{array}\right], \mathcal{C}=\left[\begin{array}{c}
2 \\
0 \\
1 \\
0 \\
1
\end{array}\right]
$$

and

$$
\mathcal{D}=\left[\begin{array}{lllll}
0 & 0 & 0 & 0 & 1 \\
0 & 0 & 0 & 1 & 0
\end{array}\right]
$$

It is clear that $\operatorname{det}(s \mathcal{E}-\mathcal{A}) \neq 0$. Using the matrices

$$
Q=\left[\begin{array}{ccccc}
1 / 2 & 0 & 0 & 0 & 0 \\
0 & 1 & 0 & 0 & 0 \\
0 & 0 & 0 & 1 / 3 & 0 \\
0 & 0 & 0 & 0 & 1 \\
0 & 0 & 1 & 0 & 0
\end{array}\right], P=\left[\begin{array}{ccccc}
1 / 2 & 0 & 0 & 0 & 0 \\
0 & 1 & 0 & 0 & 0 \\
0 & 0 & 1 & 0 & 0 \\
0 & 0 & 0 & 1 & 0 \\
0 & 0 & 0 & 0 & 1
\end{array}\right]
$$


the matrix $\mathcal{E}$ is positive equivalent to the matrix

$$
\mathcal{M}_{3}=\left[\begin{array}{ccc}
1 / 2 & 0 & 0 \\
0 & 1 & 0 \\
0 & 0 & 4 / 3
\end{array}\right] .
$$

Furthermore, we have

$$
Q \mathcal{B}=\left[\begin{array}{cc}
-1 / 4 & 0 \\
0 & 0 \\
0 & 0 \\
2 & 0 \\
0 & 1
\end{array}\right], \mathcal{D} P=\left[\begin{array}{lllll}
0 & 0 & 0 & 0 & 1 \\
0 & 0 & 0 & 1 & 0
\end{array}\right]
$$

and

$$
Q \mathcal{A} P=\left[\begin{array}{ccccc}
3 / 2 & 3 / 2 & 1 & 1 / 2 & 1 / 2 \\
3 / 2 & 1 & 2 & 1 & 1 \\
1 / 6 & 1 / 3 & 5 / 3 & 1 / 3 & 1 / 3 \\
1 / 2 & 1 & 3 & 0 & 1 \\
1 / 2 & 1 & 0 & 4 & 1
\end{array}\right] \in \mathbb{R}_{+}^{5 \times 5}
$$

Observe that

$$
\begin{aligned}
\left(\mathcal{B}_{2} \mathcal{B}_{2}^{\top} \mathcal{D}_{2}^{\top} \mathcal{D}_{2}\right)^{-1} & =\left(\begin{array}{cc}
\frac{1}{4} & 0 \\
0 & 1
\end{array}\right) \in \mathbb{R}_{+}^{2 \times 2}, \mathcal{B}_{1} \mathcal{B}_{2}^{\top} \mathcal{D}_{2}^{\top} \mathcal{D}_{2}=\left(\begin{array}{cc}
-\frac{1}{2} & 0 \\
0 & 0 \\
0 & 0
\end{array}\right) \in \mathbb{R}_{-}^{3 \times 2}, \\
\mathcal{A}_{2}+\mathcal{B}_{1} \mathcal{B}_{2}^{\top} \mathcal{D}_{2}^{\top} \mathcal{D}_{2} & =\left(\begin{array}{cc}
0 & \frac{1}{2} \\
1 & 1 \\
\frac{1}{3} & \frac{1}{3}
\end{array}\right) \in \mathbb{R}_{+}^{3 \times 2}, \mathcal{A}_{4}+\mathcal{B}_{2} \mathcal{B}_{2}^{\top} \mathcal{D}_{2}^{\top} \mathcal{D}_{2}=\left(\begin{array}{ll}
4 & 1 \\
4 & 2
\end{array}\right) \in \mathbb{R}_{+}^{2 \times 2} .
\end{aligned}
$$

Therefore, using

$$
\mathcal{K}=\left[\begin{array}{ll}
0 & 2 \\
1 & 0
\end{array}\right]
$$

we have

$$
(\mathcal{E}+\mathcal{B K} \mathcal{K})^{-1}=\left[\begin{array}{ccccc}
1 / 2 & 0 & 0 & 0 & 1 / 8 \\
0 & 1 & 0 & 0 & 0 \\
0 & 0 & 0 & 1 / 4 & 0 \\
0 & 0 & 0 & 0 & 1 / 4 \\
0 & 0 & 1 & 0 & 0
\end{array}\right] \text { and }(\mathcal{A}+\mathcal{B K} \mathcal{K} \mathcal{D})=\left[\begin{array}{ccccc}
6 & 3 & 2 & 0 & 1 \\
3 & 1 & 2 & 1 & 1 \\
1 & 1 & 0 & 4 & 2 \\
1 & 1 & 5 & 1 & 1 \\
1 & 1 & 3 & 4 & 1
\end{array}\right]
$$

that shown the system (11) can be normalized positively.

\section{Conclusion}

We have already established a sufficient condition in order for the discrete descriptor system under disturbance can be normalized positively using the difference output feedback. The conditions are generated using the concept of positive equivalent for two square matrices. 


\section{Acknowledgments}

The work was supported by Andalas University under Grant KRP1GB-PDU-Unand-2018 No. 04/UN.16.17/PP.RGB/LPPM/2018.

\section{References}

[1] Bruell, T. Explicit solutions of regular linear discrete-time descriptor systems with constant coefficients. Electronic Journal of Linear Algebra. 2009. 18: 317-338.

[2] Duan, G. R. and Wu, A. G. Impulse elimination via state feedback in descriptor linear systems. Dynamics of Continuous, Discrete and Impulsive Systems A. 2005. 3: 722-729.

[3] Li, J., Teng, Y., Zhang, Q., Jinghao, L. and Qiao, L. Eliminating impulse for descriptor system by derivative output feedback. Journal of Applied Mathematics. 2014. 2014: 1-15.

[4] Virnik, E. Stability analysis of positive descriptor systems. Linear Algebra and Its Applications. 2008. 429: 2640-2659.

[5] Kaczorek, T. Positive 1D and 2D systems. London: Springer. 2002.

[6] Muhafzan and Zulakmal. Impulse elimination for positive singular systems using derivative output feedback. Journal of Physics: Conference Series. 2017. 890: 1-4. 